TRANSACTIONS OF THE

AMERICAN MATHEMATICAL SOCIETY

Volume 350, Number 1, January 1998, Pages 101-117

S 0002-9947(98)01986-2

\title{
WEIGHTED ERGODIC THEOREMS FOR MEAN ERGODIC $L_{1}$-CONTRACTIONS
}

\author{
DOĞAN ÇÖMEZ, MICHAEL LIN, AND JAMES OLSEN \\ Dedicated to the Memory of Professor Robert Sine
}

\begin{abstract}
It is shown that any bounded weight sequence which is good for all probability preserving transformations (a universally good weight) is also a good weight for any $L_{1}$-contraction with mean ergodic (ME) modulus, and for any positive contraction of $L_{p}$ with $1<p<\infty$. We extend the return times theorem by proving that if $S$ is a Dunford-Schwartz operator (not necessarily positive) on a Lebesgue space, then for any $g$ bounded measurable $\left\{S^{n} g(\omega)\right\}$ is a universally good weight for a.e. $\omega$. We prove that if a bounded sequence has "Fourier coefficents", then its weighted averages for any $L_{1}$-contraction with mean ergodic modulus converge in $L_{1}$-norm. In order to produce weights, good for weighted ergodic theorems for $L_{1}$-contractions with quasi-ME modulus (i.e., so that the modulus has a positive fixed point supported on its conservative part), we show that the modulus of the tensor product of $L_{1}$ contractions is the product of their moduli, and that the tensor product of positive quasi-ME $L_{1}$-contractions is quasi-ME.
\end{abstract}

\section{INTRODUCTION}

Let $(X, F, \mu)$ be a $\sigma$-finite measure space, $T: L_{1}(X) \longrightarrow L_{1}(X)$ be a linear contraction, and $\tau$ be its linear modulus [CK]. $T^{*}$ will denote the adjoint of $T$. The average $\frac{1}{n} \sum_{k=0}^{n-1} T^{k}$ will be denoted by $A_{n}(T)$.

Recall that $T$ is called a Dunford-Schwartz $(D S)$ contraction if it is an $L_{1}-L_{\infty^{-}}$ contraction, and a mean ergodic operator (or $M E$ operator) if $A_{n}(T) h$ converges in $L_{1}$-norm for all $h \in L_{1}[\mathrm{Kr}]$.

Definition. We will call a contraction $T$ quasi-mean ergodic (quasi-ME) if there exists $h \in L_{1}(X)$ such that $T h=h$ and $|h|>0$ a.e. on $C_{\tau}$, the conservative part of $\tau=|T|$. An ME positive contraction is quasi-ME, but $T=-I$ shows that $T$ may be ME (and even its modulus is), without being quasi-ME. It is well-known that a DS contraction on a probability space is ME; in fact its modulus is ME.

If the modulus of a contraction $T$ has a $\sigma$-finite subinvariant measure equivalent to $\mu$, then $T$ is equivalent to a DS contraction by a change of measure, and $A_{n}(T) f$ converges a.e. for every $f \in L_{1}(X)$. Although this convergence need not hold if $T$ is a contraction only in $L_{1}$ (or only in $L_{\infty}$ ) [Kr], it was shown in [CL], [C] that if the linear modulus $\tau$ is $\mathrm{ME}$, then $T$ is also $\mathrm{ME}$, and both $A_{n}(T) f$ and $A_{n}(\tau) f$ converge a.e. for every $f \in L_{1}(X)$. This result had been established for $T$ positive by Y. Ito

Received by the editors October 9, 1995.

1991 Mathematics Subject Classification. Primary 47A35, 28D99.

(C)1998 American Mathematical Society 
$[\mathrm{I}]$ and C.-W. Kim $[\mathrm{K}]$. It is also shown in $[\mathrm{CL}]$ that there are ME $L_{1}$-contractions whose linear modulus need not be ME.

Definition. A linear contraction $T$ on $L_{1}$ is called quasi-Dunford-Schwartz (quasiDS) if its modulus has an equivalent $\sigma$-finite invariant measure on its conservative part $C$ (i.e., if $C$ is non-null, the restriction of $T$ to $L_{1}(C)$ is equivalent to a DS contraction). Dunford-Schwartz contractions and contractions with quasi-ME modulus are quasi-DS.

Theorem A. Let $T$ be a quasi-DS contraction on $L_{1}(X)$. Then for all $f \in L_{1}$, $A_{n}(T) f$ and $A_{n}(\tau) f$ converge a.e.

Proof. Let $C$ and $D$ be the conservative and dissipative parts of the modulus $\tau$. For $f \in L_{1}(X), A_{n}(T) f \rightarrow 0$ a.e. on $D$, since $\sum_{0}^{\infty}\left|T^{k} f\right| \leq \sum_{0}^{\infty} \tau^{k}|f|<\infty$ a.e. on $D$. Since $T$ is quasi-DS, there is $0 \leq u<\infty$ with $\{u>0\}=C$ and $\tau u=u$. The convergence of $A_{n}(T) f$ a.e. on $C$ now follows from Chacon's general ratio limit theorem [Kr, p. 164].

Remarks. 1. Theorem A shows that pointwise ergodic theorems depend basically only on the behavior on the conservative part. It includes both the DunfordSchwartz theorem and the result of [CL].

2. Examples of quasi-DS contractions $T$ which are neither DS nor ME are easy to construct: starting with a conservative positive DS contraction, we add a disspative part which can enter the conservative part, but has a subset that remains in the dissipative part. Since the dissipative part is not absorbing, $T$ is not DS [Kr, p. 131]. Since the dissipative part is not totally absorbed in the conservative part, $T$ is not $\mathrm{ME}[\mathrm{Kr}, \mathrm{p} .175]$.

In this article our purpose is to study weighted ergodic theorems (i.e., sequences a for which we have a.e. convergence for every $f \in L_{1}$ of

$$
\left.A_{n}(T, \mathbf{a}) f=\frac{1}{n} \sum_{k=0}^{n-1} a_{k} T^{k} f\right)
$$

for $L_{1}$-contractions with (quasi-) $\mathrm{ME}$ modulus. Weighted ergodic theorems were previously obtained by several authors [BO],[BL],[JO2] for Dunford-Schwartz operators. In section 2 we show that universally good weight sequences are good also for any $L_{1}$-contraction with ME modulus, and deduce that they are good also for any positive contraction of $L_{p}, 1<p<\infty$. In section 3 we extend the return times theorem [B],[R] by proving that if $S$ is a Dunford-Schwartz operator (not necessarily positive) on a Lebesgue space, then for any $g$ bounded measurable $\left\{S^{n} g(\omega)\right\}$ is a universally good weight for a.e. $\omega$. We also characterize (weakly) almost periodic sequences by their generation by dynamical systems. In section 4 we show that $A_{n}(T, \mathbf{a}) f$ converges in $L_{1}$-norm for every $L_{1}$-contraction $T$ with $\mathrm{ME}$ modulus and every $f \in L_{1}$ if (and only if) for every $\lambda$ with $|\lambda|=1$ the sequence $\frac{1}{N} \sum_{k=0}^{N-1} a_{k} \bar{\lambda}^{k}$ converges. In section 5 we show that the modulus of the tensor product of $L_{1}$ contractions is the product of their moduli, and that the tensor product of positive quasi-ME contractions is quasi-ME. We then apply these results to produce several classes of weights, good for weighted ergodic theorems for contractions with quasi-ME modulus.

In studying operators in $L_{\infty}$ (like Dunford-Schwartz operators), it is often useful to have these operators induced by transition measures. The next theorem $[\mathrm{H}]$ provides such a tool. 
Theorem B. Let $(X, F, \mu)$ be a probability space. Then there exists a compact Hausdorff space $K$ with the following properties:

(i) There is an order preserving isometric algebra isomorphism $\Phi$ of $L_{\infty}(X, F, \mu)$ onto $C(K)$.

(ii) Let $\tilde{F}$ be the Baire $\sigma$-algebra in $K$, and $\tilde{\mu}=\Phi^{*-1} \mu$. Then every Bairemeasurable bounded function is equivalent to a continuous function mod- $\tilde{\mu}$, and there is an order preserving isometric algebra isomorphism $\Psi$ of $L_{\infty}(X, F, \mu)$ onto $L_{\infty}(K, \tilde{F}, \tilde{\mu})$.

(iii) Let $f_{n} \in L_{\infty}$ with $\sup _{n}\left\|f_{n}\right\|_{\infty}<\infty$. Then $f_{n} \rightarrow f$ a.e. $\mu$ if and only if $\Psi f_{n} \rightarrow \Psi f$ a.e. $\tilde{\mu}$.

(iv) If $T$ is a linear operator on $L_{\infty}(X, F, \mu)$, the operator $\tilde{T}:=\Phi T \Phi^{-1}$ is an operator in $C(K)$, given by a transition measure $Q$. If $|T|^{*}$ preserves countable additivity (i.e., $T$ is a dual of an $L_{1}(\mu)$ operator), the operator $\Psi T \Psi^{-1}$ in $L_{\infty}(K, \tilde{F}, \tilde{\mu})$ is given by the (same) transition measure $Q$.

The only part which is not proved in $[\mathrm{H}]$ is the $\mu$ a.e. convegence of $f_{n}$ if $\Psi f_{n}$ converges $\tilde{\mu}$ a.e. It can be proved by similar methods, so we omit the proof.

Remark. In order to get transition measures on the original space, one needs the measure space to be a Lebesgue space. Parts (iii) and (iv) of Theorem B allow us to assume transition measures for proving a.e. convergence in general measure spaces (with no separability conditions).

\section{Almost EVERYWhere CONVERGEnCE OF Weighted AVERAGES}

If $(X, F, \mu)$ is a probability space and $T$ is a linear operator on $L_{1}(X)$, then a sequence $\mathbf{a}=\left(a_{n}\right)$ is called a good weight for $T$ if for every $f \in L_{1}(X)$, the sequence $A_{n}(T, \mathbf{a}) f=\frac{1}{n} \sum_{k=0}^{n-1} a_{k} T^{k} f$ converges $\mu$-a.e. A sequence $\mathbf{a}$ is a universally good weight if it is a good weight for every Dunford-Schwartz operator on a probability space. If $\mathbf{a}$ is a bounded universally good weight, then $A_{n}(T, \mathbf{a}) f$ converges also in $L_{1}$-norm for every Dunford-Schwartz operator $T$ on a probability space.

Remark. A universally good weight is a good weight also for all Dunford-Schwartz operators on $\sigma$-finite measure spaces. This follows from the fact that $L_{1}(D)$ is also invariant under the modulus $\tau$ [Kr, p. 131], and the conservative part is decomposed into two invariant sets, where on one $\tau$ has an invariant probability, and on the second $A_{n}(\tau) f$ converges to zero a.e.

Theorem 2.19 in Baxter-Olsen [BO] says that if $\mathbf{a}$ is a bounded good weight for every operator induced by an invertible measure preserving point transformation, then it is a good weight for all Dunford-Schwartz operators on probability spaces, and thus for all Dunford-Schwartz operators. However, part (iv) of their proof, as written, implicitly assumes that the DS operator is defined on a Lebesgue space. First, they assume that the operator and its modulus can be given by transition measures. Theorem B allows us to obtain transition measures on another space and transfer back the convergence of the weighted averages (for bounded functions), without any additional assumptions. Secondly, their proof of the following proposition (see also [JO1]) requires a Lebesgue space. We give a proof which does not require it. Thus, the result of $[\mathrm{BO}]$ is valid for all DS operators.

Proposition 2.1. Let $S$ be a contraction on $L_{\infty}(\Omega, \Sigma, m)$ with modulus $\sigma$ such that $S$ is given by a transition measure $Q(\omega, A)$ and $\sigma$ is given by a transition probability 
$P(\omega, A)$. There exists a bi-measurable function $v$ on $\Omega \times \Omega$, with $|v| \leq 1$ a.e., such that for a.e. $\omega$

$$
S g(\omega)=\int g\left(\omega^{\prime}\right) Q\left(\omega, d \omega^{\prime}\right)=\int g\left(\omega^{\prime}\right) v\left(\omega, \omega^{\prime}\right) P\left(\omega, d \omega^{\prime}\right) .
$$

Proof. Define

$$
p(A \times B)=\int 1_{A}(\omega) P(\omega, B) d m(\omega)
$$

and

$$
q(A \times B)=\int 1_{A}(\omega) Q(\omega, B) d m(\omega) .
$$

These extend to measures on $\Sigma \otimes \Sigma$ [N, III.2], and for any $E \in \Sigma \otimes \Sigma$ we have $|q(E)| \leq p(E)$ (the collection of sets $E$ with this property contains all finite unions of disjoint rectangles, and is closed under monotone limits). Let $v=d q / d p$ be the Radon-Nikodým derivative. Then $v$ is $\Sigma \otimes \Sigma$-measurable, and $|v| \leq 1 \quad m \times m$ almost everywhere, so we may assume everywhere. Fix $B \in \Sigma$. Then for every $A \in \Sigma$ we have

$$
\begin{aligned}
\int_{A} Q(\omega, B) d m(\omega) & =q(A \times B)=\iint_{A \times B} v d p \\
& =\int_{A}\left[\int 1_{B}\left(\omega^{\prime}\right) v\left(\omega, \omega^{\prime}\right) P\left(\omega, d \omega^{\prime}\right)\right] d m(\omega) .
\end{aligned}
$$

Since this holds for every $A$, we have that $Q(\omega, B)=\int 1_{B}\left(\omega^{\prime}\right) v\left(\omega, \omega^{\prime}\right) P\left(\omega, d \omega^{\prime}\right)$ for a.e. $\omega$ (the null set depending on $B$ ). Since the approximation of $g$ bounded measurable by simple functions uses only countably many sets, the claim follows.

Theorem 2.19 of $[\mathrm{BO}]$ for general measure spaces now follows from Proposition 2.1 and Theorem B, and will be used in the next proof.

Proposition 2.2. Let $\mathbf{a}=\left(a_{n}\right)_{n \geq 0}$ be a bounded universally good weight. Then the shifted sequence $\theta \mathbf{a}=\left(a_{n+1}\right)_{n \geq 0}$ is also a universally good weight.

Proof. Let $\psi$ be an invertible measure preserving transformation on a probability space $(X, F, \mu)$, and $T f(x)=f(\psi x)$. Since $\mathbf{a}$ is good for $T$, so is $\theta \mathbf{a}$, since

$$
\begin{aligned}
A_{n}(T, \theta \mathbf{a}) f & =\frac{1}{n} \sum_{k=0}^{n-1} a_{k+1} T^{k} f=\frac{1}{n} \sum_{k=0}^{n-1} a_{k+1} T^{k+1} T^{-1} f \\
& =\frac{n+1}{n}\left[A_{n+1}(T, \mathbf{a}) T^{-1} f-\frac{a_{0}}{n+1} T^{-1} f\right] .
\end{aligned}
$$

By Theorem 2.19 of [BO], $\theta \mathbf{a}$ is a universally good weight.

It has been proved that uniform sequences [BK], bounded Besicovitch sequences $[\mathrm{R}-\mathrm{N}]$, and certain sequences having a mean and a correlation [BL], are good weights for operators induced by invertible measure preserving transformations, and hence [BO] for all Dunford-Schwartz operators (i.e., are universally good weights). A simple direct proof that Besicovitch sequences are good for all DS operators is given in [JO2] (see also [O1]), and the limit was identified in [O2]. We note that [T], which deals with group actions, seems to be the first published use of Besicovitch functions for weighted ergodic theorems, including the identification of the limit. However, 
when applied to $\mathbf{Z}$, the results of [T] do not yield the results for Dunford-Schwartz operators.

Assani [A1] used the return times theorem [B, Appendix], $[\mathrm{R}]$ to obtain that for any positive Dunford-Schwartz operator $S$ on a Lebesgue space $(\Omega, \Sigma, m)$ and $g \in L_{\infty}(\Omega)$, for a.e. $w \in \Omega$ the sequence $\left\{S^{n} g(w)\right\}$ is a universally good weight. In the next section we'll show that positivity is not necessary.

Theorem 2.3. Let $\mathbf{a}=\left(a_{k}\right)$ be a bounded universally good weight sequence. If $T$ is an $L_{1}(X)$-contraction with $M E$ linear modulus, then for every $f \in L_{1}, A_{n}(T, \mathbf{a}) f$ converges a.e. and in $L_{1}(X)$.

Proof. Let $T$ be as in the theorem, and let $C$ and $D$ be the conservative and dissipative parts of the modulus $\tau$. Since the case that $\tau$ is dissipative is trivial $\left(\lim T^{n} f=0\right.$ a.e.), we assume $C$ non-empty. Since $A_{n}(T, \mathbf{a}) f$ converges a.e. on $D$ (just by the boundedness of $\mathbf{a}$ ), we have to prove convergence a.e. on $C$.

Now $L_{1}(C)$ is invariant under $\tau$ and $T$. Since $\tau$ is ME, by a change of measure the restrictions of $\tau$ and $T$ to $L_{1}(C)$ are Dunford-Schwartz in a probability space. Since $\mathbf{a}$ is universally good, we now have a.e. convergence on $C$ for $f \in L_{1}(C)$. Thus we have to prove a.e. convergence on $C$ for $f$ supported in $D$.

Let $0 \leq f \in L_{1}(D)$, and define $g_{j}=1_{D} \tau^{j} f$ and $h_{j}=1_{C} T^{j} f$. For $n>j$ we have

$$
\begin{aligned}
A_{n}(T, \mathbf{a}) f & =\frac{1}{n} \sum_{k=0}^{n-1} a_{k} T^{k} f \\
& =\frac{1}{n} \sum_{k=0}^{j-1} a_{k} T^{k} f+\frac{n-j}{n} A_{n-j}\left(T, \theta^{j} \mathbf{a}\right) 1_{C} T^{j} f+\frac{n-j}{n} A_{n-j}\left(T, \theta^{j} \mathbf{a}\right) 1_{D} T^{j} f .
\end{aligned}
$$

For $n, m>j$ we obtain

$$
\begin{aligned}
\left|A_{n}(T, \mathbf{a}) f-A_{m}(T, \mathbf{a}) f\right| & \\
\leq & \|\mathbf{a}\|_{\infty}\left(\frac{j}{n}+\frac{j}{m}\right) A_{j}(\tau)|f|+\left|\frac{n-j}{n} A_{n-j}\left(T, \theta^{j} \mathbf{a}\right) h_{j}-\frac{m-j}{m} A_{m-j}\left(T, \theta^{j} \mathbf{a}\right) h_{j}\right| \\
& +\|\mathbf{a}\|_{\infty}\left[A_{n-j}(\tau) g_{j}+A_{m-j}(\tau) g_{j}\right]
\end{aligned}
$$

As $n, m \rightarrow \infty$, the first term obviously converges to zero a.e., and the second term converges a.e. to 0 , since the function $h_{j}$ is supported on $C$ (and thus, for $j$ fixed, $A_{n}\left(T, \theta^{j} \mathbf{a}\right) h_{j}$ converges a.e., as $\theta^{j} \mathbf{a}$ is universally good by Proposition 2.2). Using Theorem A we conclude that a.e.

$$
\limsup _{n, m \rightarrow \infty}\left|A_{n}(T, \mathbf{a}) f-A_{m}(T, \mathbf{a}) f\right| \leq 2\|\mathbf{a}\|_{\infty} \lim _{n} A_{n}(\tau) g_{j} .
$$

Since $\tau^{* n} 1_{D} \rightarrow 0$ a.e. by Helmberg's criterion of mean ergodicity [Kr, p. 175], we obtain $\left\langle\tau^{n} f, 1_{D}\right\rangle=\left\langle f, \tau^{* n} 1_{D}\right\rangle \rightarrow 0$, so $\left\|g_{j}\right\|_{1} \rightarrow 0$ as $j \rightarrow \infty$. By Fatou's lemma we now obtain

$$
\int \limsup _{n, m \rightarrow \infty}\left|A_{n}(T, \mathbf{a}) f-A_{m}(T, \mathbf{a}) f\right| d \mu \leq 2\|\mathbf{a}\|_{\infty}\left\|g_{j}\right\|_{1} \rightarrow 0 .
$$

This shows that $A_{n}(T, \mathbf{a}) f(x)$ is a.e. a Cauchy sequence, so a.e. convergence is proved. Integrating the inequality obtained for $\left|A_{n}(T, \mathbf{a}) f-A_{m}(T, \mathbf{a}) f\right|$, we easily prove that $A_{n}(T, \mathbf{a}) f$ is Cauchy in norm, since $A_{n}\left(T, \theta^{j} \mathbf{a}\right) h_{j}$ converges in norm. 
Remark. The above proof of almost everywhere convergence works for $T$ quasi-DS if we assume that the dissipative part that can enter $C$ disappears (i.e., $D_{0}=D \cap$ $\left\{\lim _{n} \tau^{* n} 1_{C}>0\right\}$ satisfies $\tau^{* n} 1_{D_{0}} \rightarrow 0$ a.e. $)$, since $L_{1}\left(D-D_{0}\right)$ is always $\tau$-invariant [F, p. 18] (and a is a good weight for the restriction of $T$ to $L_{1}\left(C \cup\left(D-D_{0}\right)\right.$ ), because after a change of measure this restriction is Dunford-Schwartz).

Theorem 2.4. Let $\mathbf{a}=\left(a_{k}\right)$ be a bounded universally good weight sequence. If $T$ is an $L_{p}(X)$-contraction, $1<p<\infty$, such that for some positive $L_{p}(X)$-contraction $S$ we have $|T f| \leq S|f| \forall f \in L_{p}$, then for every $f \in L_{p}, A_{n}(T, \mathbf{a}) f$ converges a.e. and in $L_{p}(X)$.

Proof. We fix $1<p<\infty$. If $S$ is a positive contraction of $L_{p}$, then $S^{*}$ is a positive contraction of $L_{q}$ for $q=p /(p-1)$. If $0 \leq f \in L_{p}$ satisfies $S f=f$, then $S^{*} f^{p-1}=f^{p-1} \in L_{q}$. This shows that the maximal support of $S$-invariant functions is the same as the maximal support of $S^{*}$-invariant functions, which will be denoted by $Y$; and let $Z=X-Y$. We then have that both $L_{p}(Z)$ and $L_{p}(Y)$ are $S$-invariant subspaces, and $\left\|A_{n}(S)|f|\right\|_{p} \rightarrow 0$ if and only if $f$ is supported on $Z$.

Let $T$ and $S$ be as in the theorem, and let $X=Y \cup Z$ be the above decomposition for $S$. Let $f \in L_{p}(Z)$. By Akcoglu's pointwise ergodic theorem [K, pp. 190-191],

$$
\left|A_{n}(T, \mathbf{a}) f\right| \leq\|\mathbf{a}\|_{\infty} A_{n}(S)|f| \rightarrow 0 \quad \text { a.e. }
$$

It remains to prove convergence for functions supported in $Y$. Note that $L_{p}(Y)$ is also $T$-invariant. Let $0 \leq \phi \in L_{q}$ have support $Y$ and satisfy $S^{*} \phi=\phi$, and define $d \nu=\phi d \mu$. Then

$$
\int|S f| d \nu \leq \int S|f| d \nu=\int|f| S^{*} \phi d \mu=\int|f| d \nu,
$$

which shows that $T$ and $S$ are (extendable to) contractions of $L_{1}(Y, \nu)$. Since $T$ and $S$ are mean-ergodic in $L_{p}(Y, \mu)$, they are mean ergodic in $L_{1}(Y, \nu)$. The domination of $T$ by $S$ and mean-ergodicity of $S$ in $L_{1}(Y, \nu)$ imply that the linear modulus of $T$ in $L_{1}(Y, \nu)$ is also mean ergodic. By the previous theorem, $A_{n}(T, \mathbf{a}) f$ converges a.e. for any $f \in L_{1}(Y, \nu)$, so in particular for any $f \in L_{p}(Y, \mu)$.

By Akcoglu's theorem $\sup _{n}\left|A_{n}(T, \mathbf{a}) f\right| \in L_{p}(X)$ for $f \in L_{p}(X)$, which yields the norm convergence.

Remarks. 1. Our proof works for $T$ dominated by a positive mean-bounded operator $S$ such that $S^{*}$ has a strictly positive invariant function $\phi$.

2. For $T$ positive, the previous result was proved by Assani [A2], using a completely different method.

We now look at the problem of obtaining sequences which are good for all quasiDS contractions.

A bounded sequence $\mathbf{a}$ is called (weakly) almost periodic if its orbit $\left\{\theta^{n} \mathbf{a}\right\}$ is (weakly) conditionally compact in $l_{\infty}(\theta$ is the shift, as before). Let $H$ be the complex linear span of $\left\{\left(a_{k}\right): a_{k}=\lambda^{k}\right.$ for some $\lambda$ with $\left.|\lambda|=1\right\}$. The sequences in $H$ are called trigonometric polynomials. $B$, the $l_{\infty}$-norm closure of $H$, is precisely the space of all Bohr-almost periodic sequences [BL]. Any Bohr-almost periodic sequence is almost periodic (but the converse, true on $Z$, is false for one-sided sequences [BL]). In fact, we have that the space $A P$ of almost periodic sequences is the direct sum of $c_{0}$ (the space of sequences converging to 0 ) and $B$ (this classical 
result can be seen as a corollary of the Jacobs-deLeeuw-Glicksberg decomposition $[\mathrm{Kr}]$ of $A P$ induced by $\theta$ ).

Theorem 2.5. Let $\left(a_{k}\right)_{k \geq 0}$ be an almost periodic sequence. Then for every quasi$D S$ contraction $T$ on $L_{1}(X)$ and every $f \in L_{1}(X)$, the sequence $\frac{1}{n} \sum_{k=0}^{n-1} a_{k} T^{k} f(x)$ converges a.e.

Proof. Fix $T$ as in the theorem, with $\tau$ its modulus. Let $\lambda$ be in the unit circle and $f \in L_{1}(X)$. Apply Theorem A to $\lambda T$, which also has modulus $\tau$, to obtain that $\frac{1}{n} \sum_{k=0}^{n-1} \lambda^{k} T^{k} f(x)$ converges for a.e. $x$. By linearity, we see that for all $f \in L_{1}(X)$ and for all $\mathbf{a} \in H, \lim A_{n}(T, \mathbf{a}) f(x)=\lim \frac{1}{n} \sum_{k=0}^{n-1} a_{k} T^{k} f(x)$ exists for a.e. $x$. It is easy to show now that any sequence in the $l_{\infty}$-norm closure of $H$ is good for $T$.

To complete the proof, we show that any $\mathbf{a} \in c_{0}$ is good for $T$. Fix $\epsilon>0$. For $n>N$ we have $\left|a_{n}\right|<\epsilon$. Hence

$$
\begin{gathered}
\left|A_{n}(T, \mathbf{a}) f\right|=\left|\frac{1}{n} \sum_{k=0}^{n-1} a_{k} T^{k} f\right| \leq \frac{1}{n} \sum_{k=0}^{N}\left|a_{k}\right| \tau^{k}|f|+\frac{1}{n} \sum_{k=N+1}^{n-1}\left|a_{k}\right| \tau^{k}|f| \\
\leq \frac{1}{n} \sum_{k=0}^{N}\left|a_{k}\right| \tau^{k}|f|+\frac{\epsilon}{n} \sum_{k=N+1}^{n-1}\left|\tau^{k}\right| f\left|\leq \frac{1}{n} \sum_{k=0}^{N}\right| a_{k}\left|\tau^{k}\right| f\left|+\epsilon A_{n}(\tau)\right| f \mid .
\end{gathered}
$$

Using Theorem A, we obtain $\limsup _{n}\left|A_{n}(T, \mathbf{a}) f\right| \leq \epsilon \lim _{n} A_{n}(\tau)|f|$ a.e. As $\epsilon$ was arbitrary, $\lim _{n} A_{n}(T, \mathbf{a}) f=0$ a.e.

We have not been able to extend Theorem 2.5 to all weakly almost periodic sequences. In section 4 we produce some weakly almost periodic sequences which are good for every contraction with quasi-ME modulus.

\section{Generation of Bounded universally GOOd Weight SEQuenCES}

In this section we study the generation of different types of universally good bounded sequences. The return times result [B, appendix] yields that if $\psi$ is a measure preserving transformation of a Lebesgue space $(\Omega, \Sigma, m)$ and $g \in L_{\infty}(\Omega)$, then for a.e. $w \in \Omega$, the dynamically generated sequence $\left\{g\left(\psi^{n} w\right)\right\}$ is good for all operators induced by probability preserving transformations (see also $[R]$ ), and is therefore a universally good weight. We use this result to extend it to DunfordSchwartz operators on probability spaces, without the positivity assumption made by Assani [A1].

Proposition 3.1. Let $(\Omega, \Sigma, m)$ be a probability space, and $\left\{g_{n}(\omega)\right\}$ a sequence of bounded measurable functions with $\left|g_{n}(\omega)\right| \leq K$ a.e. for every $n$. If for almost every $\omega$ the sequence $\left\{g_{n}(\omega)\right\}$ is good for a (every) Dunford-Schwartz operator $T$, then the sequence $a_{n}=\int g_{n}(\omega) d m(\omega)$ is good for (every) $T$.

Proof. Let $T$ be Dunford-Schwartz on $L_{1}(X, \mu)$. Fix $f \in L_{1}(X, \mu)$. For a.e. $\omega$ we have $\mu$-a.e. convergence of $H_{N}(w, x)=\frac{1}{N} \sum_{k=1}^{N} g_{k}(\omega) T^{k} f(x)$, so by Fubini's theorem $\left(g_{n}(\omega) T^{n} f(x)\right.$ is bi-measurable) $H_{N}$ converges $m \times \mu$ a.e. Hence for a.e. $x \quad H_{N}(\omega, x)$ converges $m$-a.e., and also $f^{*}(x)=\sup _{N \geq 1} A_{N}(\tau)|f|(x)<\infty$ by the ergodic theorem. For such $x$ we have also $\left|H_{N}(\omega, x)\right| \leq K \frac{1}{N} \Sigma_{k=1}^{N} \tau^{k}|f|(x) \leq K f^{*}(x) m$-a.e., so by Lebesgue's theorem $\int H_{N}(\omega, x) d m=\frac{1}{N} \sum_{k=1}^{N} \int g_{k}(\omega) d m T^{k} f(x)$ converges. 
Theorem 3.2. Let $(\Omega, \Sigma, m)$ be a Lebesgue space, and let $S$ be a Dunford-Schwartz operator on $L_{1}(\Omega, \Sigma, m)$. For $g \in L_{\infty}(\Omega)$ there exists a null set $\Omega_{0}$ such that for any $\omega \notin \Omega_{0}$ the sequence $\left\{S^{n} g(\omega)\right\}$ is a universally good weight.

Proof. We first prove the theorem for $S$ positive with $S 1=1$. Since $(\Omega, \Sigma, m)$ is a Lebesgue space, its non-atomic part is pointwise isomorphic to the unit interval with Lebesgue measure [Ro, §2.4]. After removing a set of measure 0 and using that isomorphism, we may assume that there exists a transition probability $P(\omega, E)$ such that $S g(\omega)=\int g\left(\omega^{\prime}\right) P\left(\omega, d \omega^{\prime}\right)$ a.e. for any bounded measurable $g$ [N, p. 192]. Let $\theta$ be the Markov shift for $P$, defined on $\tilde{\Omega}=\Omega^{N}$, and denote by $P_{\omega}($.) the probability on $\tilde{\Omega}$ of the chain starting at $\omega$. Since $m$ is invariant for $P$, the probability $\tilde{m}(\cdot)=\int P_{\omega}(\cdot) d m(\omega)$, which corresponds to the chain with initial distribution $m$, is invariant for $\theta$. We also have $S^{n} g(\omega)=\int \tilde{g}\left(\theta^{n} \tilde{\omega}\right) P_{\omega}(d \tilde{\omega})$, with $\tilde{g}(\tilde{\omega})=g\left(\omega_{0}\right)$ for $\tilde{\omega}=\left\{\omega_{j}\right\}$. The invariance of $m$ implies that $(\tilde{\Omega}, \tilde{m})$ is also a Lebesgue space [Ro, §3.4]. By the return times theorem [B] (or [R]), there is $E \subset \tilde{\Omega}$ with $\tilde{m}(E)=0$ such that for $\tilde{\omega} \notin E$ the sequence $\left\{\tilde{g}\left(\theta^{n} \tilde{\omega}\right)\right\}$ is a universally good weight sequence. Since $\tilde{m}(E)=\int P_{\omega}(E) d m(\omega)$, there is $\Omega_{0} \subset \Omega$ with $m\left(\Omega_{0}\right)=0$, such that $P_{\omega}(E)=0$ for $\omega \notin \Omega_{0}$. For such $\omega$, the sequence $\left\{S^{n} g(\omega)\right\}$ is universally good by Proposition 3.1 (applied to the probability space $\left(\tilde{\Omega}, P_{\omega}\right)$ ).

We now prove the theorem for $S$ positive. We then have $S 1 \leq 1$, and $m$ is a subinvariant measure for $S$. Let $C$ and $D$ be the conservative and dissipative parts of $S$. Then both $L_{1}(C)$ and $L_{1}(D)$ are invariant under $S$ [K, p. 131], so we may assume either $m(D)=0$ or $m(C)=0$. In the first case $S 1=1$, and the previous part of the proof applies. In the second case, since $m$ is finite, $\Sigma_{n=0}^{\infty} S^{n} g(\omega)<\infty$ a.e, so $S^{n} g(\omega) \rightarrow 0$ a.e, so it is a universally good sequence.

The proof of the general case uses the construction of [BO] (see [JO1] for more details), and needs the following lemma.

Lemma 3.3. Let $(\Gamma, \lambda)$ be the unit circle with Lebesgue measure, and let $\theta$ be measure preserving in a probability space $(\Omega, \Sigma, m)$. Let $r(\omega), \phi(\omega)$ be measurable functions on $\Omega$, with $0 \leq r \leq 1$. Then the operator $R$ defined on $L_{1}(\Omega \times \Gamma, m \times \lambda)$ by $R g(\omega, \gamma)=r(\omega) g\left(\theta \omega, e^{i \phi(\omega)} \gamma\right)$ is a positive Dunford-Schwartz operator.

Proof. Clearly $R$ is positive, and $R 1(\omega, \gamma)=r(\omega) \leq 1$, so $R$ is a contraction of $L_{\infty}(\Omega \times \Gamma, m \times \lambda)$. For $0 \leq g \in L_{1}(\Omega \times \Gamma, m \times \lambda)$ we have

$$
\begin{aligned}
\|R g\|_{1} & =\int_{\Omega} r(\omega)\left[\int_{\Gamma} g\left(\theta \omega, e^{i \phi(\omega)} \gamma\right) d \lambda\right] d m(\omega) \leq \int_{\Omega}\left[\int_{\Gamma} g\left(\theta \omega, e^{i \phi(\omega)} \gamma\right) d \lambda\right] d m(\omega) \\
& =\int_{\Omega}\left[\int_{\Gamma} g(\theta \omega, \gamma) d \lambda\right] d m(\omega)=\int_{\Gamma}\left[\int_{\Omega} g(\theta \omega, \gamma) d m(\omega)\right] d \lambda \\
& =\int_{\Gamma}\left[\int_{\Omega} g(\omega, \gamma) d m(\omega)\right] d \lambda=\|g\|_{1} . \quad \square
\end{aligned}
$$

Continuation of the proof of Theorem 3.2. Let $\sigma$ be the modulus of $S$. By the argument for the positive case, we have to prove the theorem only when $\sigma$ is conservative, in which case $\sigma 1=1$, and again we assume (after removing a set of measure 0 ) that $\sigma$ is given by a transition probability $P$, and $S$ is given by a transition measure $Q$. We now obtain $v$ satisfying the properties in Proposition 2.1 .

Next we use the construction of the Markov shift for $P$ as before. For $\tilde{\omega}=\left\{\omega_{j}\right\} \in$ $\tilde{\Omega}$ we define $u(\tilde{\omega})=v\left(\omega_{0}, \omega_{1}\right)$. As before, for $g$ bounded measurable on $\Omega$ we define 
$\tilde{g}(\tilde{\omega})=g\left(\omega_{0}\right)$. We then obtain (see [JO1]) that for every $n>0$ and a.e. $\omega$

$$
S^{n} g(\omega)=\int \tilde{g}\left(\theta^{n} \tilde{\omega}\right) \prod_{j=0}^{n-1} u\left(\theta^{j} \tilde{\omega}\right) d P_{\omega} .
$$

Let $r(\tilde{\omega})=|u(\tilde{\omega})|$, and $\phi=\arg (r / u)$ where $u \neq 0$, with $\phi=0$ if $u=0$. Define $R$ on $L_{1}(\tilde{\Omega} \times \Gamma, m \times \lambda)$ by

$$
R h(\tilde{\omega}, \gamma)=r(\tilde{\omega}) h\left(\theta \tilde{\omega}, e^{i \phi(\tilde{\omega})} \gamma\right)
$$

By the lemma, $R$ is a positive Dunford-Schwartz operator. For $g(\omega)$ bounded measurable on $\Omega$ we take $h(\tilde{\omega}, \gamma)=\gamma \tilde{g}(\tilde{\omega})$. By induction we obtain

$$
R^{n} h(\tilde{\omega}, \gamma)=\left[\prod_{j=0}^{n-1} r\left(\theta^{j} \tilde{\omega}\right)\right] \tilde{g}\left(\theta^{n} \tilde{\omega}\right) \gamma\left[\prod_{j=0}^{n-1} e^{i \phi\left(\theta^{j} \tilde{\omega}\right)}\right]=\gamma \tilde{g}\left(\theta^{n} \tilde{\omega}\right) \prod_{j=0}^{n-1} u\left(\theta^{j} \tilde{\omega}\right)
$$

Since $R$ is a positive Dunford-Schwartz operator, the first part of the proof yields that for $\tilde{m} \times \lambda$ almost every $(\tilde{\omega}, \gamma)$, the sequence $\left\{R^{n} h\right\}$ is a universally good weight. Hence for a.e. $\gamma$, the sequence $\gamma \tilde{g}\left(\theta^{n} \tilde{\omega}\right) \prod_{j=0}^{n-1} u\left(\theta^{j} \tilde{\omega}\right)$ is a universally good weight for $\tilde{m}$ almost every $\tilde{\omega}$. By choosing one such $\gamma$, we have that $\tilde{g}\left(\theta^{n} \tilde{\omega}\right) \prod_{j=0}^{n-1} u\left(\theta^{j} \tilde{\omega}\right)$ is a universally good weight for $\tilde{m}$ almost every $\tilde{\omega}$. Hence it is a universally good weight $P_{\omega}$-a.e. for $m$ almost every $\omega$. The formula for $S^{n} g(\omega)$ and Proposition 3.1 yield that for such $\omega$ the sequence $S^{n} g(\omega)$ is a universally good weight.

Remark. Theorem B allows us to assume transition probabilities on the representation space so that almost everywhere convergence of norm-bounded sequences of $L_{\infty}$ functions is preserved. However, since we want to prove another property of $\left\{S^{n} g(\omega)\right\}$ (i.e., being good), we need that the transition measures $P$ and $Q$ be obtained on the space $\Omega$ itself, which is why we need at the very beginning to assume that $(\Omega, \Sigma, m)$ is a Lebesgue space.

We next look at the generation of (weakly) almost periodic sequences. Note that the Fourier coefficients of a complex measure on the unit circle form a weakly almost periodic sequence [Bu, p. 37]. Weakly almost periodic sequences are Besicovitch, but the converse is false (e.g., [LO]). A dynamical model for generating Besicovitch sequences is given in $[\mathrm{BL}]$.

Definition. A vector $x$ in a Banach space $B$ is (weakly) almost periodic for a linear contraction $T$ on $B$ if the orbit $\left\{T^{n} x\right\}$ is (weakly) conditionally compact. If every vector is (weakly) almost periodic for $T$, we call $T$ (weakly) almost periodic.

Thus, a bounded sequence $\mathbf{a}$ is (weakly) almost periodic if it is (weakly) almost periodic for the shift $\theta$ in $l_{\infty}$.

Theorem 3.4 [Bu, p. 36]. Let $T$ be a contraction in a Banach space B, and let $x$ be a weakly almost periodic vector for $T$. Then for any $x^{*} \in B^{*}$, the sequence $a_{k}=\left\langle T^{k} x, x^{*}\right\rangle$ is weakly almost periodic. If $x$ is almost periodic, $\left(a_{k}\right)$ is almost periodic.

Theorem 3.5. Let a be a (weakly) almost periodic sequence. Then there exists a continuous self-map $\psi$ of a compact metric space $\Omega$ such that $S f=f \circ \psi$ is (weakly) almost periodic on $C(\Omega)$, and $a_{k}=g\left(\psi^{k} w\right)$ for some $g \in C(\Omega)$ and $w \in \Omega$. 
Proof. Note first that every weakly almost periodic sequence a can be obtained as in the previous theorem $[\mathrm{E}]$, by taking $T=\theta$ on $l_{\infty}, x=\mathbf{a}$, and $x^{*}=(1,0,0, \ldots) \in l_{\infty}^{*}$.

We may assume that $B$ is the closed linear manifold generated by the orbit of $x$. Then $B$ is a separable Banach space invariant under $T$, and thus $\Omega$, the unit ball of $B^{*}$, is a compact metric space in the weak-* topology. $\psi$, the restriction of $T^{*}$ to $\Omega$, is a continuous map, and we define $S f=f \circ \psi$ on $C(\Omega)$.

Let $n_{j}$ be a sequence of positive integers. Then there is a subsequence $r_{i}=n_{j_{i}}$ with $T^{r_{i}} x$ converging weakly in $B$. Clearly $T^{r_{i}} y$ converges weakly in $B$ for any $y \in B$. Denoting $f_{y}\left(y^{*}\right)=\left\langle y, y^{*}\right\rangle$, we obtain that $S^{r_{i}} f_{y}$ converges pointwise to a continuous function. Clearly $\left\{f \in C(\Omega): S^{r_{i}} f\right.$ converges pointwise to a continuous function $\}$ is a closed subalgebra which separates points and contains the constants (and is self-adjoint in the complex case), so it is $C(\Omega)$. Hence $S$ is weakly almost periodic, since weak convergence in $C(\Omega)$ is equivalent to pointwise convergence to a continuous limit. If $x$ is almost periodic for $T$, then the above arguments show that $S$ is almost periodic.

If $g\left(y^{*}\right)=\left\langle x, y^{*}\right\rangle$ on $\Omega$, and $w=x^{*}$, then $a_{k}=g\left(T^{* k} x^{*}\right)=g\left(\psi^{k} w\right)$.

Remark. The previous theorem gives a precise characterization of the generation of (weakly) almost periodic sequences, since by Theorem 3.4, every sequence produced by $\psi$ as in Theorem 3.5 is (weakly) almost periodic.

\section{NORM CONVERGENCE OF WEIGHTED AVERAGES}

Theorem 2.3 shows that if $\mathbf{a}$ is a universally good weight sequence and $T$ is a contraction of $L_{1}$ with mean ergodic modulus, then the averages $A_{n}(T, \mathbf{a}) f$ converge also in norm for every $f \in L_{1}$. This raises the question: for which sequences a do the weighted averages $A_{n}(T, \mathbf{a}) f$ converge in norm for every contraction $T$ of $L_{1}$ with ME modulus and every $f \in L_{1}$ ? A complete solution (for bounded sequences) is obtained in Theorem 4.2.

We want to deal here also with certain unbounded sequences. Following [Be] (where the definition is made for $\mathbf{R}$ ), we define for $1 \leq p<\infty$ the set $W_{p}$ of complex sequences,

$$
W_{p}=\left\{\mathbf{a}=\left(a_{n}\right): \limsup _{N \rightarrow \infty} \frac{1}{N} \sum_{n=0}^{N-1}\left|a_{n}\right|^{p}<\infty\right\} .
$$

$W_{p}$ clearly contains all bounded sequences. On $W_{p}$ we have a semi-norm (the p-semi-norm), defined by $\|\mathbf{a}\|_{W_{p}}^{p}=\lim \sup _{N \rightarrow \infty} \frac{1}{N} \sum_{k=0}^{N-1}\left|a_{k}\right|^{p}$.

Definition. A sequence $\mathbf{a}=\left(a_{n}\right)$ has Fourier coefficients if for every $\lambda$ with $|\lambda|=1$, the sequence $\frac{1}{N} \sum_{k=0}^{N-1} \bar{\lambda}^{k} a_{k}$ converges as $N \rightarrow \infty$, say to $C(\lambda)$. We then call the function $C(\lambda)$ the Fourier coefficient function of $\mathbf{a}$.

Theorem 4.1. Let $1<p<\infty$, and let $\mathbf{a}=\left(a_{n}\right)$ be a sequence in $W_{p}$ which has Fourier coefficients. Then for every weakly almost periodic operator $T$ in a Banach space $B,\left\{A_{n}(T, \mathbf{a})\right\}$ converges in the strong operator topology.

Proof. By the Jacobs-deLeeuw-Glicksberg decomposition [Kr, p. 105] we have $B=$ $B_{0} \oplus B_{1}$, with

$B_{1}=$ closed linear manifold generated by $\{u \in B: T u=\lambda u$ for some $|\lambda|=1\}$, 


$$
B_{0}=\left\{w \in B: \frac{1}{N} \sum_{k=0}^{N-1}\left|\left\langle T^{k} w, v^{*}\right\rangle\right| \rightarrow 0 \quad \forall v^{*} \in B^{*}\right\} .
$$

The existence of Fourier coefficients implies the norm convergence of $A_{n}(T, \mathbf{a}) u$ for $u$ with $T u=\lambda u$. Since $T$ is weakly almost periodic, it is power-bounded. Since $W_{p} \subset W_{1}$, for every $n$ we have $\left\|A_{n}(T, \mathbf{a})\right\| \leq \sup _{j}\left\|T^{j}\right\| \sup _{N} \frac{1}{N} \sum_{k=0}^{N-1}\left|a_{k}\right|$. Hence we have strong convergence on $B_{1}$.

Since $T$ is power-bounded, by a change of norm we may take $\|T\| \leq 1$. Let $q=p /(p-1)$ be the dual index. In order to prove the convergence for $w \in B_{0}$, we use the arguments of Jones and Lin [Kr, p. 109], with some modification since $\mathbf{a}$ is not assumed bounded. On $\Omega$, the unit ball of $B^{*}$ (with the weak-* topology), we define a continuous map $\psi\left(v^{*}\right)=T^{*} v^{*}$, and a continuous function $f\left(v^{*}\right)=\left|\left\langle w, v^{*}\right\rangle\right|^{q}$. For each $v^{*} \in \Omega$ we have that $f\left(\psi^{n} v^{*}\right)$ converges to zero along a subsequence of density 1 (depending on $v^{*}$ ), so $\frac{1}{N} \sum_{k=0}^{N-1} f\left(\psi^{k} v^{*}\right) \rightarrow 0$ for any $v^{*} \in \Omega$. This yields weak convergence to 0 of $\frac{1}{N} \sum_{k=0}^{N-1} f \circ \psi^{k}$, and hence strong convergence by the mean ergodic theorem. Thus,

$$
\sup _{v^{*} \in \Omega} \frac{1}{N} \sum_{k=0}^{N-1}\left|\left\langle T^{k} w, v^{*}\right\rangle\right|^{q}=\sup _{v^{*} \in \Omega} \frac{1}{N} \sum_{k=0}^{N-1} f\left(\psi^{k} v^{*}\right) \rightarrow 0 .
$$

Now for $w \in B_{0}$ we use Hölder's inequality to obtain

$$
\begin{gathered}
\left\|A_{n}(T, \mathbf{a}) w\right\|=\sup _{v^{*} \in \Omega}\left|\frac{1}{n} \sum_{k=0}^{n-1}\left\langle a_{k} T^{k} w, v^{*}\right\rangle\right| \\
\leq \sup _{v^{*} \in \Omega}\left(\frac{1}{n} \sum_{k=0}^{n-1}\left|a_{k}\right|^{p}\right)^{1 / p}\left(\frac{1}{n} \sum_{k=0}^{n-1}\left|\left\langle T^{k} w, v^{*}\right\rangle\right|^{q}\right)^{1 / q} \rightarrow 0 .
\end{gathered}
$$

Theorem 4.2. Let $1<p<\infty$. The following are equivalent for $\mathbf{a} \in W_{p}$ :

(i) The sequence a has Fourier coefficients.

(ii) For every weakly almost periodic operator $T$ in a Banach space, $\left\{A_{n}(T, \mathbf{a})\right\}$ converges in the strong operator topology.

(iii) For every contraction $T$ in $L_{1}$ with mean ergodic modulus, $\left\{A_{n}(T, \mathbf{a}) f\right\}$ converges strongly for every $f \in L_{1}$.

(iv) For every contraction $T$ in $L_{1}$ induced by an ergodic probability preserving transformation, $\left\{A_{n}(T, \mathbf{a}) f\right\}$ converges strongly for every $f \in L_{1}$.

Proof. (iii) trivially implies (iv), and (iv) implies (i) by taking, for a given $\lambda$ of modulus 1 , the rotation by $\lambda$ on the unit circle (and $f(z)=\bar{z}$ ). (i) $\Rightarrow$ (ii) is Theorem 4.1 .

We prove (ii) $\Rightarrow$ (iii). We first observe that a Dunford-Schwartz operator $T$ in a finite measure space is weakly almost periodic: For $f \in L_{2}$ the sequence $\left\{T^{n} f\right\}$ is weakly conditionally compact in $L_{2}$, hence in $L_{1}$. A standard approximation argument will finish the proof.

Let $T$ be a contraction of $L_{1}(X, F, \mu)$ with modulus $\tau$ which is ME. If $\tau$ is dissipative, then $\left\|T^{n} f\right\| \leq\left\|\tau^{n}|f|\right\| \rightarrow 0$ for any $f \in L_{1}$, so $T$ is obviously almost periodic, and Theorem 4.1 applies. Thus, we assume that $C$, the conservative part of $\tau$, has positive measure. The restrictions of $\tau$ and $T$ to $L_{1}(C)$ are Dunford-Schwartz after a change of (finite) measure, since $\tau$ has a finite invariant measure supported 
on $C$, by mean ergodicity. Hence $A_{n}(T, \mathbf{a}) f$ converges in norm for $f \in L_{1}(C)$ by Theorem 4.1. Now the convergence has to be proved only for $f$ supported in $D$, and we proceed as in the proof of Theorem 2.3: define $g_{j}=1_{D} \tau^{j}|f|$ and $h_{j}=1_{C} T^{j} f$. For $n>j$ we have

$A_{n}(T, \mathbf{a}) f=\frac{1}{n} \sum_{k=0}^{j-1} a_{k} T^{k} f+\frac{n-j}{n} A_{n-j}\left(T, \theta^{j} \mathbf{a}\right) 1_{C} T^{j} f+\frac{n-j}{n} A_{n-j}\left(T, \theta^{j} \mathbf{a}\right) 1_{D} T^{j} f$.

Since $\mathbf{a} \in W_{p} \subset W_{1}$, we have $\sup _{n} \frac{1}{n} \sum_{k=0}^{n-1}\left|a_{k}\right|<\infty$, and we denote this supremum by $\alpha$. Hence (all norms are $L_{1}$-norms)

$$
\begin{gathered}
\left\|\frac{1}{n} \sum_{k=0}^{j-1} a_{k} T^{k} f\right\| \leq \frac{j}{n} \alpha\|f\|, \\
\left\|\frac{n-j}{n} A_{n-j}\left(T, \theta^{j} \mathbf{a}\right) 1_{D} T^{j} f\right\| \leq\left\|\frac{1}{n} \sum_{k=j}^{n-1}\left|a_{k}\right| \tau^{k-j} g_{j}\right\| \leq \alpha\left\|g_{j}\right\| .
\end{gathered}
$$

For fixed $j$ we have $h_{j} \in L_{1}(C)$, and $\theta^{j} \mathbf{a} \in W_{p}$. By applying Theorem 4.1 to the restriction of $T$ to $L_{1}(C)$, we obtain

$$
\limsup _{n, m \rightarrow \infty}\left\|A_{n}(T, \mathbf{a}) f-A_{m}(T, \mathbf{a}) f\right\| \leq 2 \alpha\left\|g_{j}\right\| \rightarrow_{j \rightarrow \infty} 0 .
$$

Hence $A_{n}(T, \mathbf{a}) f$ is a Cauchy sequence in $L_{1}$, and therefore converges.

We do not know if Theorem 4.1 is true for $p=1$ - our proof clearly fails for $p=1$. However, we can use the method of $[\mathrm{T}]$ to obtain the following result for certain sequences of $W_{1}$.

Theorem 4.3. Let $1<p_{j}<\infty$, and let $\mathbf{a}^{(j)} \in W_{p_{j}}$ have Fourier coefficients. Let $\mathbf{a} \in W_{1}$ satisfy $\lim _{j}\left\|\mathbf{a}-\mathbf{a}^{(j)}\right\|_{W_{1}}=0$. Then $\mathbf{a}$ satisfies all properties (i)-(iv) of Theorem 4.2. Moreover, for a given $T$ as in Theorem 4.2, the limit operators satisfy

$$
\left\|\lim _{n \rightarrow \infty} A_{n}(T, \mathbf{a})-\lim _{n \rightarrow \infty} A_{n}\left(T, \mathbf{a}^{(j)}\right)\right\| \leq\left\|\mathbf{a}-\mathbf{a}^{(j)}\right\|_{W_{1}} \sup _{k}\left\|T^{k}\right\| .
$$

We omit the proof of convergence of $A_{n}(T, \mathbf{a})$, since it is similar to [T]. Let $\sup _{j}\left\|T^{j}\right\|=C$ and let $L$ and $L^{(j)}$ be the limits of $A_{n}(T, \mathbf{a})$ and $A_{n}\left(T, \mathbf{a}^{(j)}\right)$. The inequality for the limits follows from

$$
\begin{gathered}
\left\|\left(L-L^{(j)}\right) v\right\|=\left\|\lim _{N} \frac{1}{N} \sum_{k=0}^{N-1}\left[a_{k}-a_{k}^{(j)}\right] T^{k} v\right\|=\lim _{N}\left\|\frac{1}{N} \sum_{k=0}^{N-1}\left[a_{k}-a_{k}^{(j)}\right] T^{k} v\right\| \\
\leq \limsup _{N \rightarrow \infty} \frac{1}{N} \sum_{k=0}^{N-1}\left|a_{k}-a_{k}^{(j)}\right| C\|v\|=C\|v\|\left\|\mathbf{a}-\mathbf{a}^{(j)}\right\|_{W_{1}} .
\end{gathered}
$$

Recall that we have defined $H$ to be the complex linear span of $\left\{\mathbf{a}=\left(a_{k}\right)\right.$ : $a_{k}=\lambda^{k}$ for some $\lambda$ with $\left.|\lambda|=1\right\}$. For $1 \leq p<\infty$, the set $B_{p}$ of $p$-Besicovitch sequences is defined as the closure of $H$ in the $p$-semi-norm. Since sequences in $H$ have Fourier coefficients, Theorem 4.3 yields the norm convergence properties of 1-Besicovitch sequences (not necessarily bounded). However, for a given $T$ the proof of Theorem 4.3 uses just the strong convergence of $A_{n}\left(T, \mathbf{a}^{(j)}\right)$ for each $j$, so we have the following stronger result. 
Theorem 4.4. Let $\mathbf{a}$ be a 1-Besicovitch sequence, and let $T$ be a power-bounded linear operator on a Banach space such that $\lambda T$ is $M E$ for every $|\lambda|=1$. Then $A_{n}(T, \mathbf{a})$ converges in the strong operator topology.

Since $\lambda T$ is mean ergodic for all $\lambda,|\lambda|=1$, the convergence of $L^{(j)} v$ in the proof of Theorem 4.3 is immediate. The limit can be approximated by the limits obtained for the approximating trigonometric polynomials, using the inequality in Theorem 4.3, which applies also here, with the same proof. For $\mathbf{a} \in B_{2}$ the limit was identified in [T] (for group actions), and later in [O2] (in the present set-up).

Remark. An example of a universally good bounded sequence (which then has Fourier coefficients) that is not in $B_{1}$ is given in [BL, p.323].

\section{5. $L_{1}$-CONTRACTIONS OF PRODUCT SPACES WITH APPLICATIONS TO WEIGHTED AVERAGES}

Let $(X, F, \mu)$ and $(Y, \Sigma, m)$ be $\sigma$-finite measure spaces and let $T$ and $S$ be contractions on $L_{1}(X)$ and $L_{1}(Y)$, respectively. By $\left(X^{\prime}, F^{\prime}, \mu^{\prime}\right)$ we will denote the product measure space $(X \times Y, F \times \Sigma, \mu \times m)$. Similarly, the map $V: L_{1}\left(X^{\prime}\right) \longrightarrow L_{1}\left(X^{\prime}\right)$ will denote the (tensor) product operator $T \otimes S$. Clearly $V$ is a linear $L_{1}\left(X^{\prime}\right)$ contraction. In the sequel, $T$ will always denote a linear contraction on $L_{1}(X)$ and $S$ will denote a linear contraction on $L_{1}(Y)$. Their linear moduli will be denoted by $\tau$ and $\sigma$, respectively.

Theorem 5.1. Let $T$ and $S$ be $L_{1}(X)$ - and $L_{1}(Y)$-contractions, respectively, and let $\tau$ and $\sigma$, respectively, be their linear moduli. Then the linear modulus of $T \otimes S$ is $\tau \otimes \sigma$.

Proof. Denote $V=T \otimes S$. Then $\nu$, the linear modulus of $V$, is the unique positive $L_{1}\left(X^{\prime}\right)$-contraction such that $\|T \otimes S\|=\|\nu\|$ and $|(T \otimes S) f| \leq \nu|f|$ for all $f \in$ $L_{1}\left(X^{\prime}\right)$. Moreover $[\mathrm{CK}]$, for $f \in L_{1}^{+}\left(X^{\prime}\right)$,

$$
\nu f(x, w)=\sup \left\{|(T \otimes S) g(x, w)|: g \in L_{1}\left(X^{\prime}\right),|g| \leq f\right\} .
$$

Obviously $\tau \otimes \sigma$ is a positive $L_{1}\left(X^{\prime}\right)$-contraction, and clearly $\nu \leq \tau \otimes \sigma$. Hence, it is enough to show that $\tau \otimes \sigma \leq \nu$.

Let $f=h \otimes g$, where $h$ and $g$ are positive and are finite linear combinations of characteristic functions in $L_{1}(X)$ and $L_{1}(Y)$, respectively. Then

$$
\begin{aligned}
& (\tau \otimes \\
& \quad=\sup \left\{|T u|: u \in L_{1}(X),|u| \leq h\right\} \sup \left\{|S v|: v \in L_{1}(Y),|v| \leq g\right\} \\
& \quad=\sup \left\{|(T u) \otimes(S v)|: u \otimes v \in L_{1}\left(X^{\prime}\right),|u| \leq h,|v| \leq g\right\} \\
& \quad=\sup \left\{|(T \otimes S)(u \otimes v)|: u \otimes v \in L_{1}\left(X^{\prime}\right),|u| \leq h,|v| \leq g\right\} \\
& \quad \leq \sup \left\{|(T \otimes S)(u \otimes v)|: u \otimes v \in L_{1}\left(X^{\prime}\right),|u \otimes v| \leq h \otimes g\right\} \\
& \quad \leq \sup \left\{|(T \otimes S) z|: z \in L_{1}\left(X^{\prime}\right),|z| \leq h \otimes g\right\}=\nu f .
\end{aligned}
$$

Since such functions are dense in $L_{1}^{+}\left(X^{\prime}\right)$, it follows that $(\tau \otimes \sigma) f \leq \nu f$, for all $f \in L_{1}^{+}\left(X^{\prime}\right)$, by the continuity of the operators $\tau \otimes \sigma$ and $\nu$. .

Let $T$ be a ME operator on $L_{1}(X)$. Then by the splitting theorem of Yosida $[\mathrm{Kr}]$, we have $L_{1}(X)=F_{T} \oplus \operatorname{cl}\left(N_{T}\right)$, where $F_{T}=\left\{g \in L_{1}(X): T g=g\right\}$ and $N_{T}=\left\{g-T g: g \in L_{1}(X)\right\}$. Note that $F_{T}$ is a closed subspace. 
Theorem 5.2. Let $\tau$ and $\sigma$ be positive quasi mean ergodic contractions in $L_{1}(X)$ and $L_{1}(Y)$, respectively. Then $\nu=\tau \otimes \sigma$ is quasi mean ergodic, and $C_{\tau} \times C_{\sigma}=C_{\nu}$. If $\tau$ and $\sigma$ are mean ergodic, so is $\nu$.

Proof. We first show that we always have (even without the quasi mean ergodicity assumptions) the inclusion $C_{\tau} \times C_{\sigma} \supset C_{\nu}$. By [Kr, Theorem 3.1.3] there exists a sequence $D_{j} \subset X, D_{j} \uparrow D_{\tau}$, such that

$$
\sum_{n=0}^{\infty} \tau^{* n} 1_{D_{j}} \in L_{\infty}(X)
$$

Since $\sigma^{* n} 1_{Y} \leq 1$, we have

$$
\sum_{n=0}^{\infty} \nu^{* n}\left(1_{D_{j}} \otimes 1_{Y}\right)=\sum_{n=0}^{\infty} \tau^{* n} 1_{D_{j}} \otimes \sigma^{* n} 1_{Y} \in L_{\infty}\left(X^{\prime}\right) .
$$

Hence $D_{j} \times Y \subset D_{\nu}$; therefore $D_{\tau} \times Y \subset D_{\nu}$. By symmetry, $X \times D_{\sigma} \subset D_{\nu}$. Therefore,

$$
C_{\nu} \subset\left[\left(X \times D_{\sigma}\right) \cup\left(D_{\tau} \times Y\right)\right]^{c}=C_{\tau} \times C_{\sigma} .
$$

Now, assume $\tau$ and $\sigma$ are quasi-ME and let $0 \leq g \in L_{1}(X)$ with $\tau g=g$, $\{g>0\}=C_{\tau}$, and $0 \leq h \in L_{1}(Y), \sigma h=h,\{h>0\}=C_{\sigma}$ (which exist by quasi$\mathrm{ME})$. Then

$$
\nu(g \otimes h)=\tau g \otimes \sigma h=g \otimes h
$$

yields a $\nu$-invariant function in $L_{1}\left(X^{\prime}\right)$, supported on $C_{\tau} \times C_{\sigma}$. Hence $C_{\tau} \times C_{\sigma} \subset C_{\nu}$, and the equality holds.

Assume now that $\tau$ and $\sigma$ are ME. Since $\tau^{* n} 1_{D_{\tau}} \downarrow 0$ a.e. and $\sigma^{* n} 1_{D_{\sigma}} \downarrow 0$ a.e. by Helmberg's criterion, we have $\nu^{* n} 1_{D_{\nu}}(x, y) \leq \tau^{* n} 1_{D_{\tau}}(x)+\sigma^{* n} 1_{D_{\sigma}}(y) \rightarrow 0$ a.e. on $X^{\prime}$. Hence $\nu$ is ME.

Remark. Let $X=Z^{2}$ and $Y=Z$ ( $Z$ denotes the set of integers). By taking $\tau$ the simple symmetric random walk on $X$ and $\sigma$ the simple symmetric random walk on $Y$, we have $C_{\tau} \times C_{\sigma}=X \times Y$. However, $\nu$ is a (symmetric) random walk on $Z^{3}$, so is transient, and hence $C_{\nu}=\emptyset$.

Theorem 5.3. Let $T$ and $S$ be contractions in $L_{1}(X)$ and $L_{1}(Y)$, respectively. If their moduli $\tau$ and $\sigma$ are quasi-ME, so is $\nu$, the modulus of $V=T \otimes S$, and both $A_{n}(\nu) f$ and $A_{n}(V) f$ converge a.e. for all $f \in L_{1}\left(X^{\prime}\right)$.

Proof. By Theorem 5.1, the linear modulus of $V$ is $\nu=\tau \otimes \sigma$, so $\nu$ is quasi-ME by Theorem 5.2. The a.e convergence follows from Theorem A (see [CL]).

A similar proof yields the following.

Corollary 5.4. Let $T$ and $S$ be as in Theorem 5.3. If $\tau$ and $\sigma$ are mean ergodic, so are $\nu$ and $V$, and both $A_{n}(V) f$ and $A_{n}(\nu) f$ converge a.e. for all $f \in L_{1}\left(X^{\prime}\right)$.

Remark. If $S$ is induced by a measure preserving transformation on a probability space $Y$, then $S=\sigma$ and is a conservative mean ergodic contraction (actually a positive Dunford-Schwartz contraction).

We now use the previous results to obtain good weight sequences. 
Theorem 5.5. Let $(\Omega, \Sigma, m)$ be a probability space and $S$ be a Dunford-Schwartz contraction on $L_{1}(\Omega)$. Let $g \in L_{\infty}(\Omega), h \in L_{1}(\Omega)$, and define $a_{k}=\left\langle S^{k} g, h\right\rangle$. Then for every contraction $T$ with quasi-ME linear modulus $\tau$ on $L_{1}(X)$ and $f \in L_{1}(X)$, the sequence $\frac{1}{n} \sum_{k=0}^{n-1} a_{k} T^{k} f(x)$ converges a.e. If $\tau$ is $M E, L_{1}$-convergence also holds.

Proof. Since $m$ is finite, $\sigma=|S|$ is mean ergodic. By Theorem 5.2, $\tau \otimes \sigma$ is quasiME, so

$$
\frac{1}{n} \sum_{k=0}^{n-1}(T \otimes S)^{k}(f \otimes g)(x, w)=\frac{1}{n} \sum_{k=0}^{n-1} T^{k} f(x) S^{k} g(w)
$$

converge $\mu \times m$-a.e., say to $\eta(x, w)$. As $\tau$ is quasi-ME, $f^{*}(x)=\sup _{n} \frac{1}{n} \sum_{k=0}^{n-1} \tau^{k}|f|(x)$ is finite a.e. on $X$ by Theorem A. Hence,

$$
\left|\frac{1}{n} \sum_{k=0}^{n-1} T^{k} f(x) S^{k} g(w)\right| \leq\|g\|_{\infty} f^{*}(x)
$$

$\mu \times m$-a.e. on $X \times \Omega$, so for a.e. $x$, the inequality holds for $m$-a.e. $w$. Fixing such an $x$, one obtains from Lebesgue's bounded convergence theorem that

$$
\begin{aligned}
A_{n}(T, \mathbf{a}) f(x)=\frac{1}{n} \sum_{k=0}^{n-1} a_{k} T^{k} f(x)= & \int \frac{1}{n} \sum_{k=0}^{n-1} T^{k} f(x) S^{k} g(w) h(w) m(d w) \\
& \longrightarrow \int \eta(x, w) h d m .
\end{aligned}
$$

Now let $h \in L_{\infty}(\Omega)$. If $\tau$ is ME, then $\tau \otimes \sigma$ and $T \otimes S$ are ME, and the convergence to $\eta(x, w)$ is also in $L_{1}(\mu \times m)$-norm (and $\eta \in L_{1}(X \times \Omega)$ ). Hence for such an $h$ we have

$$
\begin{gathered}
\int\left|A_{n}(T, \mathbf{a}) f(x)-\int h(w) \eta(x, w) m(d w)\right| \mu(d x) \\
\leq \iint\left|\frac{1}{n} \sum_{k=0}^{n-1} S^{k} g(w) h(w) T^{k} f(x)-\eta(x, w) h(w)\right| m(d w) \mu(d x) \\
\leq\|h\|_{\infty}\left\|A_{n}(T \times S)(f \otimes g)-\eta\right\|_{L_{1}(\mu \times m)} \longrightarrow 0 .
\end{gathered}
$$

If $h \in L_{1}(\Omega)$, the norm convergence of $A_{n}(T, \mathbf{a}) f$ is proved by standard approximation arguments.

Remark. The sequence a defined in the previous theorem is weakly almost periodic (by Theorem 3.4 for $h \in L_{2}$, and then by standard approximations), so the norm convergence when $\tau$ is ME can also be deduced from Theorem 2.3. The novelty in the previous theorem (compared with Theorem 2.3) is that a.e. convergence is obtained without requiring $\tau$ to be ME.

If the sequence $\mathbf{a}$ is bounded and $T$ is quasi-DS then, for every $f \in L_{1}(X)$, Theorem A yields

$$
\sup _{n}\left|\frac{1}{n} \sum_{k=0}^{n-1} a_{k} T^{k} f\right| \leq\|\mathbf{a}\|_{\infty} \sup _{n} \frac{1}{n} \sum_{k=0}^{n-1} \tau^{k}|f|<\infty .
$$

In that case, by the Banach principle, $\mathbf{a}$ is a good weight for $T$ if we can show the a.e. convergence of $A_{n}(T, \mathbf{a}) f$ for $f$ in a dense subspace of $L_{1}(X)$. 
Theorem 5.6. Let $(\Omega, \Sigma, m)$ be a probability space, $S$ a Dunford-Schwartz contraction on $L_{1}(\Omega)$, and $g \in L_{\infty}(\Omega)$. If $(X, F, \mu)$ is a probability space with $L_{1}(X)$ separable, and $T$ is an $L_{1}(X)$-contraction with quasi-mean ergodic modulus $\tau$, then for a.e. $w \in \Omega,\left\{S^{n} g(w)\right\}$ is good for $T$ (and $\tau$ ).

Proof. Consider the product space $X^{\prime}=X \times \Omega$, let $V=T \otimes S$, and let $\sigma$ be the linear modulus of $S$. By Theorem 5.1, $\nu=\tau \otimes \sigma$ is the modulus of $V$, and, by Theorem 5.3, for all $f \in L_{1}(X)$, both $A_{n}(V) h$ and $A_{n}(\nu) h$ converge $\mu^{\prime}$-a.e., where $h=f \otimes g \in L_{1}\left(X^{\prime}\right)$.

Let $f_{j}$ be a countable dense sequence in $L_{1}(X)$. Then the convergence obtained above for $A_{n}(V)\left(f_{j} \otimes g\right)$ yields that, for a.e. $w \in \Omega$,

$$
\frac{1}{n} \sum_{k=0}^{n-1} S^{k} g(w) T^{k} f_{j}
$$

converges $\mu$-a.e. for each $f_{j}$. Hence for such $w$ we have a.e. convergence for a dense subset of $L_{1}(X)$, and (by the Banach principle) the sequence $\left\{S^{n} g(w)\right\}$ is good for $T$. Similarly, it is good for $\tau$.

Remark. In our proof of the previous theorem, the null-set in $\Omega$ depends on $T$. Theorem 3.2 yields that for a.e. $\omega$ the sequence $\left\{S^{n} g(\omega)\right\}$ is a universally good weight sequence.

\section{ACKNOWLEDGEMENTS}

1. The second and third authors are grateful for the hospitality and support of Northwestern University, where part of this research was carried out.

2. The second author is also grateful for the hospitality and support of North Dakota State University.

\section{REFERENCES}

[A1] I. Assani, The return times and the Wiener-Wintner property for mean bounded positive operators in $L^{p}$, Ergodic Th. \& Dynam. Sys., 12 (1992), 1-12. MR 93d:47017

[A2] I. Assani, Universal weights from dynamical systems to mean-bounded positive operators on $L_{p}$, Proceedings of the Conf. on A.E. Conv. in Ergodic Th. and Prob.-II, Academic Press, 1991, 9-16. MR 92i:47007

[B] J. Bourgain, Pointwise ergodic theorems for arithmetic sets, with an appendix on return time sequences jointly with H. Furstenberg, Y.Katznelson and D.Ornstein, Inst. Hautes Etudes Sci. Publ. Math., 69 (1989), 5-45. MR 90k:28030

[BK] A. Brunel and M. Keane, Ergodic theorems for operator sequences, Z. Wahr. Verw. Gebiete, 12 (1969), 231-240. MR 42:3831

[BL] A. Bellow and V. Losert, The weighted pointwise ergodic theorem and the individual ergodic theorem along subsequences, Trans. AMS, 288 (1985), 307-345. MR 86c:28035

[BO] J. R. Baxter and J. Olsen, Weighted and subsequential ergodic theorems, Canadian J. Math., 35 (1983), 145-166. MR 84g:47005

[Be] A.S. Besicovitch, Almost periodic functions, Cambridge University Press, London, 1932 (reprinted Dover, 1954). MR 16:817a

[Bu] R.B. Burckel, Weakly almost periodic functions on semigroups, Gordon and Breach, NewYork, 1970. MR 41:8562

[CK] R.V. Chacon and U. Krengel, Linear modulus of a linear operator, Proc. AMS, 15 (1964), 553-559. MR 29:1543

[C] D. Çömez, A generalization of Dunford-Schwartz theorem, Turkish J. Math, 15 (1991), 29-34. MR 92e: 47011 
[CL] D. Çömez and M. Lin, Mean ergodicity of $L_{1}$-contractions and pointwise ergodic theorems, Proceedings of the Conf. on A.E. Conv., in Ergodic Th. and Prob.-II, Academic Press, 1991, 113-126. MR 92k:47016

[E] W. F. Eberlein, Abstract ergodic theorems and weak almost periodic functions, Trans. AMS, 67 (1949), 217-240. MR 12:112a

[F] S. Foguel, The ergodic theory of Markov processes, Van Nostrand, New York, 1969. MR 41:6299

$[\mathrm{H}] \quad$ S. Horowitz, Transition probabilities and contractions of $L_{\infty}, \mathrm{Z}$. Wahrscheinlichkeitstheorie verw. Geb. 24 (1972), 263-274. MR 48:9849

[I] Y. Ito, Uniform integrability and the pointwise ergodic theorem, Proc. AMS, 16 (1965), 222-227. MR 30:2121

[JO1] R. Jones and J. Olsen, Subsequence pointwise ergodic theorems for operators in $L_{p}$, Israel J. Math., 77 (1992), 33-54. MR 94d:47008

[JO2] R. Jones and J. Olsen, Multiparameter weighted ergodic theorems, Canadian J. Math., 46 (1994), 343-356. MR 95g:47020

[K] C.W. Kim, A generalization of Ito's theorem concerning the pointwise ergodic theorem, Ann. of Math. Stat., 39 (1968), 214-218. MR 38:2276

[Kr] U. Krengel, Ergodic Theorems, de Gruyter, Berlin, 1985. MR 87i:28001

[LO] M. Lin and J. Olsen, Besicovitch functions and weighted ergodic theorems for LCA group actions, Convergence in Ergodic Theory and Probability (V. Bergelson, P. March, J. Rosenblatt, eds.), de Gruyter, Berlin - New York, 1996, 277-289. CMP 97:02

[N] J. Neveu, Mathematical foundations of the calculus of probability, Holden-Day, SanFrancisco, 1965. MR 33:6660

[O1] J. Olsen, The individual weighted ergodic theorem for bounded Besicovitch sequences, Canad. Math. Bull., 25 (1982), 468-471. MR 84b:47013

[O2] J. Olsen, Calculation of the limit in the return times theorem for Dunford-Schwartz operators, Proc. Alexandria (Egypt) conference on "Ergodic Theory and its Connections With Harmonic Analysis," London Mathematical Society Lecture Note Series \#205, Cambridge University Press, London, 1995, 359-368. MR 96f:47021

[R] D. Rudolph, A joinings proof of Bourgain's return time theorem, Ergodic Th. \& Dynam. Sys., 14 (1994), 197-203. MR 95e:28015

[Ro] V. A. Rohlin, On the fundamental ideas of measure theory, Mat. Sbornik (NS) 25(67) (1949), 107-150. English translation: AMS Translations, Series One, 10 (1962), 1-54. MR 11:18f

[R-N] C. Ryll-Nardzewski, Topics in ergodic theory, Probability - Winter School (Karpacz, 1975), Springer-Verlag Lecture Notes in Math., 472 (1975), 131-156. MR 52:11003

[T] A. Tempelman, Ergodic theorems for amplitude modulated homogeneous random fields, Litovsk. Mat. Sb. 14 (1974), no. 4, 221-229 (in Russian). English trans. in Lithuanian Math. Trans. 14 (1975), 698-704. MR 53:1709

Department of Mathematics, North Dakota State University, Fargo, North Dakota 58105

Department of Mathematics, Ben Gurion University of the Negev, Beer Sheva, Israel

Department of Mathematics, North Dakota State University, Fargo, North Dakota 58105 